\title{
circCD151 promotes GLI2 expression by regulating miR-30d-5p and enhancing proliferation, invasion and stemness of lung cancer
}

\author{
LIHONG ZHAO, HONG ZHENG and PING JIANG
}

Department of Respiratory and Critical Care Medicine, Tianjin First Central Hospital, Tianjin 300110, P.R. China

Received December 15, 2020; Accepted May 28, 2021

DOI: $10.3892 / \mathrm{mmr} .2021 .12338$

\begin{abstract}
To investigate the changes of circular (circ)RNA circCD151 expression in lung cancer tissues and cells and its effects on proliferation, migration and invasion of lung cancer cells. The relative expression levels of circCD151 in lung cancer tissues and lung cancer cells (A549 and NCI-H292) were determined by reverse transcription-quantitative PCR. The effects of silencing or upregulation of circCD151 on the activity and clonal forming ability of A549 and NCI-H292 cells were detected by CCK- 8 and cloning formation experiments. Transwell invasion assay detected the effects of silencing or upregulation of circCD151 on the migration and invasion ability of A549 and NCI-H292 cells. The regulatory effect of circCD151 on miR-30d-5p was detected by dual luciferase reporter gene. The relative expression level of circCD151 in lung cancer tissues was significantly higher compared with that in adjacent tissues. The relative expression level of circCD151 in A549 and NCI-H292 cells was significantly higher compared with that in human lung epithelial cells. In A549 and NCI-H292 cells, silencing circCD151 decreased cell activity and clonal formation ability and invasion ability was also significantly decreased. circCD151 was upregulated in A549 and NCI-H292 cells and the activity and clonal formation ability of A549 and NCI-H292 cells were significantly increased and the invasion ability was also significantly increased. Double luciferase reporter assay confirmed the ceRNA regulatory mechanism of circCD151/miR-30d-5p/GLI2. In the present study, in vivo and in vitro functional studies demonstrated that circCD151 may promote the proliferation, invasion and cell stemness of lung cancer cells. Further molecular mechanism studies demonstrated that circCD151 could promote the malignant
\end{abstract}

Correspondence to: Dr Lihong Zhao, Department of Respiratory and Critical Care Medicine, Tianjin First Central Hospital, 24 Fukang Road, Nankai, Tianjin 300110, P.R. China

E-mail: zhaolhyzx@126.com

Abbreviations: NSCLC, Non-small-cell lung cancer; GLI2, Glioma cancer related gene homologous protein 2

Key words: lung cancer, circular RNAs, circCD151, proliferation, invasion, cell stemness proliferation of lung adenocarcinoma by targeting miR-30d-5p and upregulating GLI2 expression. From the perspective of circRNA, the present study will provide new clues to the pathogenesis and prognostic judgment of lung adenocarcinoma and provide a new target for clinical treatment.

\section{Introduction}

Non-small-cell lung cancer (NSCLC) is the commonest lung malignant tumor worldwide, with a high incidence and mortality rate, ranking first among all malignant tumors (1-3). Therefore, it is necessary to further explore the function of the new gene and its precise mechanism in the malignant progression of lung cancer (4-6).

Circular (circ)RNAs are special non-coding RNAs that connect the $3^{\prime}$ and $5^{\prime}$ ends to form a closed circular structure $(7,8)$. CircRNAs are abundant, conserved and stabile in the cytoplasm. Increasing evidence suggests that circRNAs may serve an important role in the occurrence and development of cancers, including lung adenocarcinoma, and may be a novel marker of cancer (9). The role of circRNA in lung cancer has been the subject of a number of studies, but as a biomarker and its mechanism of action remains to be elucidated. Jiang et al (10) demonstrated that hsa_circ_0007385 is involved in the occurrence and development of NSCLC and is expected to become a potential marker and diagnosis and treatment target. Zhang et al (11) demonstrated that hsa circ_0014130 is closely related to NSCLC and can be used as a biomarker of NSCLC. Yao et al (12) confirmed that the expression level of hsa_circ_100876 is increased in NSCLC tissues and Kaplan-Meier survival analysis demonstrated that the overall survival rate of patients with high expression of hsa_circ_100876 was shorter. The results of Wei et al (13) showed that circCD151 can promote tumor progression and immune evasion by regulating the miR-370-3p/CXCL12 axis of melanoma. However, the role of circCD151 in lung cancer has not been reported.

MicroRNAs (miRNAs/miRs) are a type of non-coding RNA with a length of 22 nt $(14,15)$, which directly degrade and inhibit protein synthesis and regulate post-transcriptional gene expression level through complete or incomplete complementary binding with target mRNA 3'UTR (16). miRNAs have been shown to regulate a variety of physiological and pathological processes, such as cell differentiation, cell proliferation and tumor formation $(17,18)$. Some miRNAs can participate 
directly in the formation of human tumors, such as lung cancer, craniocerebral tumor, liver cancer, colorectal cancer and lymphoma. miRNAs can be used as both oncogenes and tumor suppressor genes to participate in multiple signaling pathways of human tumor formation $(19,20)$. Therefore, the study of specific miRNA function provides a new direction for tumor treatment and prevention (21).

A number of studies have found that abnormal activation of the Hh signaling pathway is closely related to the occurrence of liver cancer and the maintenance of malignant biological characteristics (22-24). Glioma cancer related gene homologous protein 2 (GLI2) is one of the signal pathways of terminal transcription factors. GLI2 transfers extracellular Hh to the cell nucleus, which, combined with the downstream gene promoter regions, starts the transcription of target genes, including Gli1; the Hh signaling pathway serves a very important role $(25,26)$.

The purpose of the present study was to investigate the expression of circCD151 in lung cancer tissues and cells and its effect on proliferation, migration and invasion of lung cancer cells. The regulatory effect of circCD151 on miR-30d-5p/GLI2 axis was also investigated.

\section{Materials and methods}

Clinical patient information. Samples of 20 cases of lung adenocarcinoma and normal tissues around the carcinoma ( $>5 \mathrm{~cm}$ away from the tumor) were collected from October 2020 to January 2021. All specimens were taken from hospital thoracic surgery lobectomy and confirmed by postoperative pathology for the patients with lung adenocarcinoma ( I-III A period). There were 10 males and 10 females, aged $~ 51-78(63.11 \pm 6.31)$ years. Cancer staging was based on the American Cancer Federation's Cancer Staging Guidelines, 8 th edition $(27,28)$. The specimens were immediately frozen in liquid nitrogen and stored at $-80^{\circ} \mathrm{C}$. None of the patients had received radiation or chemotherapy before surgery and had no history of malignant tumor. The present study was approved by the medical Ethics Committee of Tianjin First Central Hospital (approval no. TJ202008096). All patients signed informed consent.

Cell culture. Human normal lung epithelial cell BEAS-2B, lung cancer cell lines A549, NCI-H460, NCI-H292, 95-D were purchased from the American Type Culture Collection. All cells were subcultured in RPMI-1640 medium (Gibco; Thermo Fisher Scientific, Inc.). RPMI-1640 was supplemented with $10 \%$ fetal bovine serum (Gibco; Thermo Fisher Scientific, Inc.), $100 \mathrm{U} / \mathrm{ml}$ penicillin and $100 \mathrm{mg} / 1$ streptomycin (Gibco; Thermo Fisher Scientific, Inc.). Human normal bronchial epithelial cells BEAS-2B were subcultured in DMEM (Gibco; Thermo Fisher Scientific, Inc.) supplemented with $10 \%$ fetal bovine serum, $100 \mathrm{U} / \mathrm{ml}$ penicillin and $100 \mathrm{mg} / \mathrm{l}$ streptomycin. Incubation was at $37^{\circ} \mathrm{C}$ and $5 \% \mathrm{CO}_{2}$. The fresh medium was changed every 2-3 days and the confluence of cells was $>75 \%$ for cell passage.

Cell transfection. Plasmid transfection was performed with Lipofectamine ${ }^{\circledR} 2000$ according to the manufacturer's protocol (Invitrogen; Thermo Fisher Scientific, Inc.). Transfection was initiated when cell confluence reached $80 \%$. A549 and
NCI-H292 cells were divided into two groups: One group was transfected with $2.5 \mu \mathrm{g}$ pcDNA.3.1 empty vector and the other was transfected with $2.5 \mu \mathrm{g}$ pcDNA.3.1 circCD151-expression vector (Guangzhou Geneseed Biotech Co., Ltd.). Following $24 \mathrm{~h}$ of transfection at $37^{\circ} \mathrm{C}$ with $5 \% \mathrm{CO}_{2}$, the cells were subcultured at a dilution ratio of 1:5. After transfection for $48 \mathrm{~h}$, the transfection efficiency was determined by reverse transcription-quantitative PCR (RT-qPCR). Follow-up experiments were performed $48 \mathrm{~h}$ after transfection.

miRNAs and small interfering RNAs (siRNAs/si) were purchased from Shanghai GenePharma Co. Ltd. RNAiMAX transfection reagent (Thermo Fisher Scientific, Inc.), and 50 pmol miR-mimics-negative control (NC; 5'-UUGUAC UACACAAAAGUACUG-3'), 50 pmol miR-30d-5p mimics (5'-UGUAAACAUCCCCGACUGGAAG-3'), 50 pmol miR-inhibitor-NC (5'-CAGUACUUUUGUGUAGUACAA-3'), 50 pmol miR-30d-5p inhibitor (5'-CUUCCAGUCGGG GAUGUUUACA-3') were mixed separately and subsequent operations were carried out according to the kit instructions to transfect miRNAs into the cells. Following $48 \mathrm{~h}$ of cell transfection, the transfection efficiency was determined using RT-qPCR and subsequent experiments were performed.

The siRNAs were transfected into cells following the same method; that is, 50 pmol si-NC (5'-UUCUCCGAACGUGUC ACGU-3'), 50 pmol si-circCD151 (5'-CACTTGTAGAGC AGAATTC-3') or 50 pmol si-GLI2 (5'-GACAUGAGCUCC AUGCUCA-3') were transfected into cells using RNAiMAX. Follow-up experiments were performed $48 \mathrm{~h}$ after transfection.

Separating the nucleus and cytoplasm. The nucleus and cytoplasm were separated using a nuclear/cytoplasmic separation kit (Thermo Fisher Scientific, Inc.). Cells $\left(5 \times 10^{6}\right)$ were taken and centrifuged at $500 \mathrm{x} \mathrm{g}$ at $4^{\circ} \mathrm{C}$ for $2-3 \mathrm{~min}$ to collect the cells. The cells were washed twice with cold PBS. Cold extract A $(200 \mu \mathrm{l})$ was added to the cell precipitation, mixed well and agitated over ice for $30 \mathrm{~min}$ then centrifuged for $5 \mathrm{~min}$ at $1,200 \mathrm{x}$ at $4^{\circ} \mathrm{C}$. The supernatant was aspirated into another clean precooled centrifuge tube to obtain the cytoplasmic component. The precipitate (nuclear component) was washed once with PBS, then centrifuged at $4^{\circ} \mathrm{C}$ for $5 \mathrm{~min}$ at $2,000 \mathrm{x} \mathrm{g}$ and the supernatant was discarded. The precipitate was resuspended with $200 \mu \mathrm{l}$ storage solution B and stored for later use or directly used for experiments.

CCK 8 experiments. A549 and NCI-H292 cell suspension was inoculated in 96-well plates with 5 multiple wells, $\sim 3,000$ cells/well/100 $\mu 1$ in each group. The culture plates were placed in an incubator for pre-culture $\left(37^{\circ} \mathrm{C}, 5 \% \mathrm{CO}_{2}\right)$. CCK8 detection reagent $(10 \mu \mathrm{l})$ was added to each well and incubated in an incubator for $4 \mathrm{~h}$ at $37^{\circ} \mathrm{C}$ with $5 \% \mathrm{CO}_{2}$. The absorbance at $450 \mathrm{~nm}$ was measured with a microplate reader and the OD value of each well was determined.

Transwell experiment. Matrigel was diluted with blank medium (1:3). The diluted Matrigel was added to the superior compartment surface of the membrane at the bottom of the Transwell compartment. Matrigel was polymerized into gel at $37^{\circ} \mathrm{C}$ for $30 \mathrm{~min}$. DMEM complete medium $(0.6 \mathrm{ml})$ containing $10 \%$ FBS was added into the Transwell chamber. The prepared transfected cell suspension $\left(1.0 \times 10^{6} / \mathrm{ml}\right)$ of 
$20 \mu 1$ was absorbed and added to $200 \mu 1$ serum-free DMEM culture. After mixing well $200 \mu \mathrm{l}$ was absorbed into the upper chamber, which was incubated for $48 \mathrm{~h}$ in an incubator at $37^{\circ} \mathrm{C}$ and $5 \% \mathrm{CO}_{2}$. Any cells that had not been passed through the upper chamber were wiped off with a cotton swab. The lower chamber was washed with PBS and fixed with $4 \%$ paraformaldehyde for $15 \mathrm{~min}$ at room temperature. After removing the fixing solution, the chamber was cleaned with PBS once and $0.1 \%$ crystal violet was used for staining at room temperature for $15 \mathrm{~min}$. Then, the staining solution was removed and the cells in the lower compartment were washed with PBS for 2-3 times and left to dry naturally at room temperature. The culture plates were placed under an inverted microscope and 3 fields of each filter membrane were randomly selected for examination and imaging.

$R T-q P C R$. Reverse transcription and RT-qPCR followed the manufacturer's instructions. Total RNA was extracted from tissues and cells using TRIzol ${ }^{\circledR}$ reagent (Thermo Fisher Scientific,Inc.). RNA was reversely transcribed into cDNA using the Prime-Script one-step RT-PCR kit (Takara Biotechnology Co., Ltd.). LightCycler ${ }^{\circledR} 480$ SYBR Green I Master mix (Roche Diagnostics) was used to detect the expression of each gene on an ABI PRISM 7300 detection system (Applied Biosystems; Thermo Fisher Scientific, Inc.). The primer sequences used for the qPCR were as follows: circCD151 forward: 5'-CACTTG TAGAGCAGAATTCTC-3', reverse: 5'-CGTTGAACTCAC CCATCCTGG-3'; miR-30d-5p forward: 5'-UGUAAACAU CCCCGACUGGAAG-3', reverse: 5'-TGTAAACATCCCCGA CTGGAAGA-3'; GLI2 forward: 5'-GAGGGATCCGCCCTC ACCTCCATCAAT-3', reverse: 5'-GAGGAATTCCTAGGT CATCATGTTCAGG-3'; SOX2 forward: 5'-GCCGATGTG AAACTTTTGTCG-3', reverse: 5'-GGCAGCGTGACTTAT CCTTCT-3; CD44 forward: 5'-TTGCAGTCAACAGTCGAA GAAG-3', reverse: 5'-CCTTGTTCACCAAATGCACCA-3'; Oct4 forward: 5'-CTTGCTGCAGAAGTGGGTGGAGGA A-3', reverse: 5'-CTGCAGTGTGGGTTTCGGGCA-3'; Nanog forward: 5'-AATACCTCAGCCTCCAGCAGATG-3', reverse: 5'-TGCGTCACACCATTGCTATTCTTC-3'; U6 forward: 5'-CTCGCTTCGGCAGCACATATACT-3', reverse: 5'-ACG CTTCACGAATTTGCGTGTC-3'; GAPDH forward: 5'-GGT ATGACAACGAATTTGGC-3', reverse 5'-GAGCACAGGG TACTTTATTG-3'. U6 and GAPDH were used as reference genes. Reverse transcription conditions were $16^{\circ} \mathrm{C}$ for $30 \mathrm{~min}$, $50^{\circ} \mathrm{C}$ for $30 \mathrm{~min}, 75^{\circ} \mathrm{C}$ for $15 \mathrm{~min}$ and $4^{\circ} \mathrm{C}$ for use. PCR conditions were $10 \mathrm{~min}$ at $95^{\circ} \mathrm{C}, 15 \mathrm{sec}$ at $95^{\circ} \mathrm{C}$ and $60 \mathrm{sec}$ at $60^{\circ} \mathrm{C}$ for 40 cycles. A total of three parallel samples were provided for each $20 \mu \mathrm{l}$ system. U6 RNA was used as the internal standard for normalization. The relative expression of gene levels were quantified using the $2^{-\Delta \Delta \mathrm{Cq}}$ method and normalized to the internal reference gene (29).

Clone formation experiment. The low soluble point agarose solution was prepared with distilled water. The temperature was maintained at $40^{\circ} \mathrm{C}$ after autoclave sterilization. The agarose was mixed with $2 \mathrm{X}$ medium in a ratio of $1: 1$. Then $3 \mathrm{ml}$ of the mixture was added to a plate with a diameter of $6 \mathrm{~cm}$. After it cooled and solidified, the agar was placed in the incubator $\left(37^{\circ} \mathrm{C}\right)$ for later use. Cells of each group were inoculated in 6 -well plates with $1 \times 10^{3}$ cells per well. Visible cell colonies were formed after $\sim 2$ weeks of culture. After washing with PBS three times and fixing with $75 \%$ ethanol for $30 \mathrm{~min}$ at room temperature, the cells were stained with $1 \%$ crystal violet for $10 \mathrm{~min}$ at room temperature. After PBS washing, images were captured using an optical microscope (Nikon Corporation) in five randomly selected fields of view Three replicates were used for each experiment and the average of the three replicates was calculated.

StarBase and TargetScan analysis. In this study, the StarBase database (http://starbase.sysu.edu.cn) was used to analyze the binding sites of circCD151 and miR-30d-5p. The TargetScan 7.2 database (http://www.targetscan.org/vert_72) was used to analyze the binding sites between miR-30d-5p and GLI2.

Double luciferase reporter gene assay. Binding sites between circCD151 and miR-30d-5p were predicted using StarBase. Binding sites between GLI2 and miR-30d-5p were predicted using TargetScan. The luciferase reporter vectors, psiCHECK2 -circCD151-wild-type (WT), psiCHECK2-circCD151-mutant (MT), psiCHECK2-GLI2-WT and psiCHECK2-GLI2-MT (all Promega Corporation), were co-transfected with miR-30d-5p mimics and miR-NC respectively using Lipofectamine. Following $48 \mathrm{~h}$ of cell transfection, the relative luciferase activity was detected using a Dual-Luciferase Reporter assay system (Promega Corporation). The ratio of the luminescence intensity of Renilla luciferase to that of firefly luciferase reflected the binding force.

Statistical analysis. The data were presented as mean \pm standard deviation and analyzed using SPSS 17.0 software (SPSS, Inc.). Each group of experiment was repeated three times. One-way ANOVA and Tukey's post hoc test was used to test the data of variables between groups. Student's t-test test was used to compare the variable data between the two groups. Paired Student's t-test was used for tumor and adjacent normal tissues. $\mathrm{P}<0.05$ was considered to indicate a statistically significant difference.

\section{Results}

circCD151 is upregulated in NSCLC. RT-qPCR results demonstrated that circCD151 was upregulated in NSCLC cell lines to different degrees compared with normal lung epithelial cell BEAS-2B (Fig. 1A, P<0.05). The expression of circCD151 was then evaluated in NSCLC tissues. The results demonstrated that the expression of circCD151 was significantly upregulated in NSCLC tissues compared with that in adjacent tissues (Fig. 1B, $\mathrm{P}<0.01$ ). These results suggest that circCD151 may serve an oncogene role in NSCLC. Further tests revealed that circCD151 was mainly expressed in the cytoplasm (Fig. 1C).

Silencing circCD151 inhibits the activity, invasion and clonal formation of NSCLC cells. Since circCD151 was upregulated in NSCLC, siRNA weas used to knock down circCD151 expression to evaluate its biological function. First, RT-qPCR results demonstrated that circCD151 siRNA inhibited A549 and NCI-H292 cells significantly (Fig. 2A and B). The results of cck- 8 demonstrated that the activity of A549 and nci-h292 cells after transfection with si-circCD151 for 

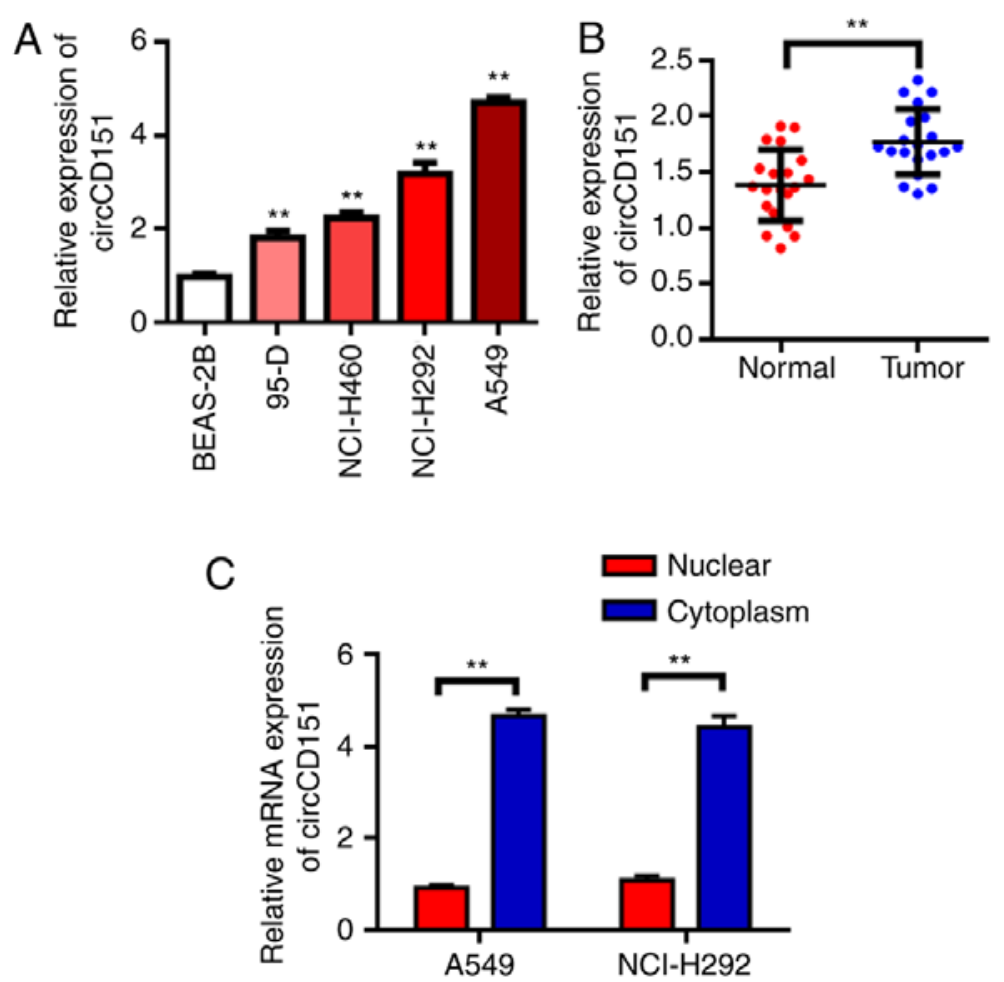

Figure 1. circCD151 is upregulated in lung cancer patients and lung cancer cell lines. (A) circCD151 was upregulated in lung cancer cell lines. (B) qPCR was used to detect the expression of circCD151 in tumor tissues and adjacent tissues of lung cancer patients. (C) Subcellular fractionation experiment to determine the cellular location of circCD151. RT-qPCR data demonstrated that circCD151 was mainly located in the cytoplasm. Data are presented as the means \pm standard deviation. ${ }^{* *} \mathrm{P}<0.01$. Circ, circular RNA; q, quantitative; RT, reverse transcription.

$72 \mathrm{~h}$ was significantly lower than that of the control group (Fig. 2C and D) $(\mathrm{P}<0.05)$. Colony formation test results demonstrated that the number of colony formation in the si-circCD151 transfection group was significantly lower than that in the control group (Fig. 2E and F; P<0.01). Transwell was further used to evaluate the ability of cell invasion. The results demonstrated that the number of invaded cells in the si-circCD151 transfection group was significantly lower than that in the control group (Fig. $2 \mathrm{G}$ and H; P<0.01). RT-qPCR was used to detect the changes in the expression levels of SOX2, CD44, OCT4 and Nanog in A549 and NCI-H292 cells after circCD151 knockdown. Results demonstrated that the expression levels of SOX2, CD44, OCT4 and Nanog were decreased in A549 and NCI-H292 cells after circCD151 knockdown (Fig. 2I-L).

circCD151 targeted regulation of miR-30d-5p expression. StarBase was used to predict that miR-30d-5p might be a target gene for circCD151; the forecast sequence is demonstrated in Fig. 3A. Results of the dual-luciferase reporter gene demonstrated that overexpression of miR-30d-5p could reduce the luciferase activity of wild-type circCD151, while co-transfection of miR-30d-5p mimics and the circCD151-MUT vector mutated at the targeted site led to loss of the inhibitory effect of miR-30d-5p on luciferase activity (Fig. 3B and C). The expression level of miR-30d-5p in lung cancer cell lines after circCD151 knockdown was detected by qPCR The results demonstrated that the knockdown of circCD151 significantly promoted the expression of miR-30d-5p, while the overexpression of circCD151 inhibited the expression of
miR-30d-5p (Fig. 3D and E). Detection results of circCD151 overexpression efficiency is presented in Fig. S1. Clinical specimens of 20 patients with lung cancer were tested and it was found that miR-30d-5p was lowly expressed in lung cancer tissues compared with the para-cancer control group (Fig. 3F). Therefore, miR-30d-5p was the direct target gene of circCD151 and circCD151 could negatively regulate the expression of miR-30d-5p.

GLI2 is the target gene of miR-30d-5p. Target genes of miR-30d-5p were predicted with TargetScan and it was found that GLI2 was the candidate target gene of miR-30d-5p (Fig. 4A). Subsequently, luciferase reporter gene validation experiments were used to find that miR-30d-5p can inhibit luciferase activity of wild type GLI2, but had no inhibitory effect on mutant GLI2 (Fig. 4B). Further investigation revealed that miR-30d-5p could negatively regulate the expression of GLI2. RT-qPCR was used to detect the effects of miR-30d-5p knockdown on the expression of GLI2 mRNA and the results demonstrated that miR-30d-5p knockdown could significantly promote the expression level of GLI2 in lung cancer cells. The overexpression of miR-30d-5p inhibited the expression of GLI2 (Fig. 4C-F). Then, 20 lung cancer patients were tested and it was found that GLI2 was highly expressed in lung cancer tissues (Fig. 4G) compared with the para-cancer control group. The above experimental results demonstrated that GLI2 was the target gene of miR-30d-5p and miR-30d-5p could negatively regulate the expression of GLI2. In addition, RT-qPCR was used to detect the changes in the expression of GLI2 in A549 and NCI-H292 cells after circCD151 knockdown. The 
A

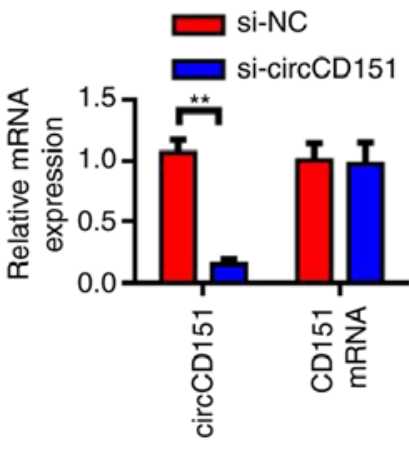

B

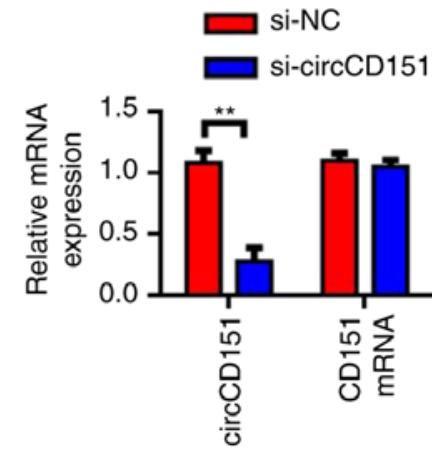

C

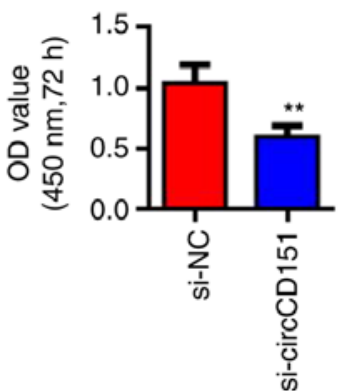

$\mathrm{NCl}-\mathrm{H} 292$

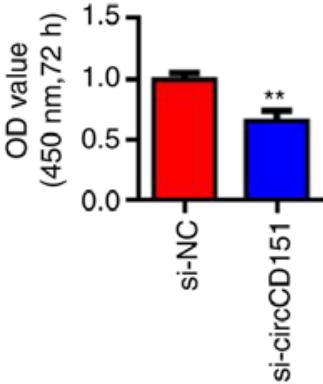

E

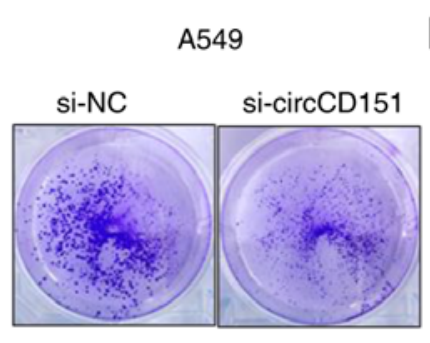

F

$\mathrm{NCl}-\mathrm{H} 292$

G

A549

$\mathrm{H}$

$\mathrm{NCl}-\mathrm{H} 292$
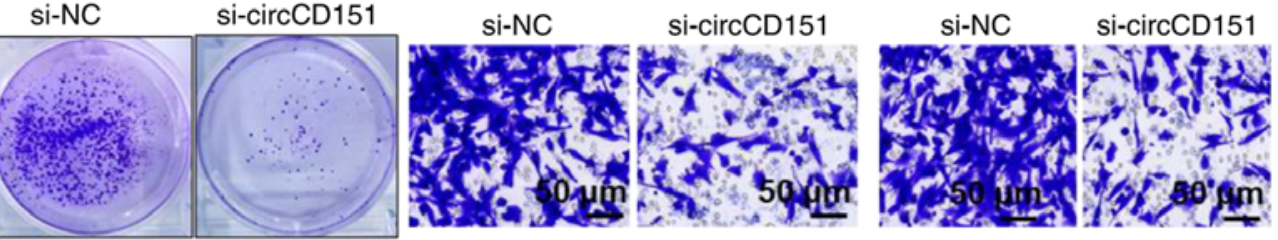

A549

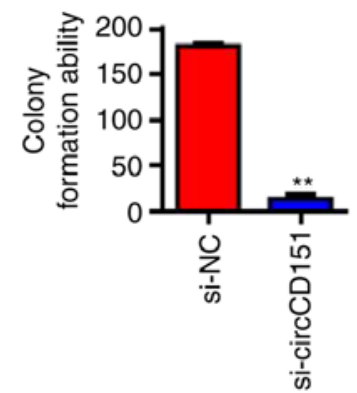

I
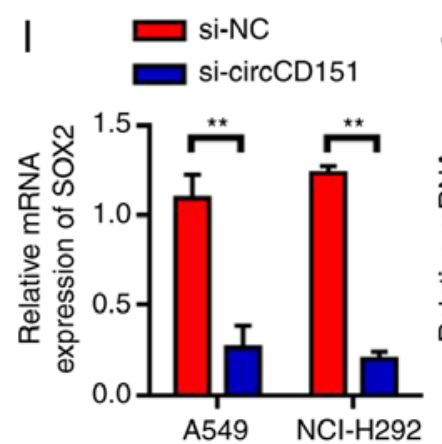

$\mathrm{NCl}-\mathrm{H} 292$

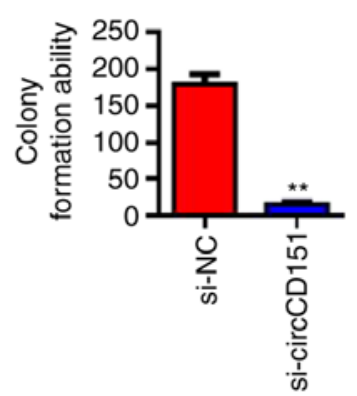

J
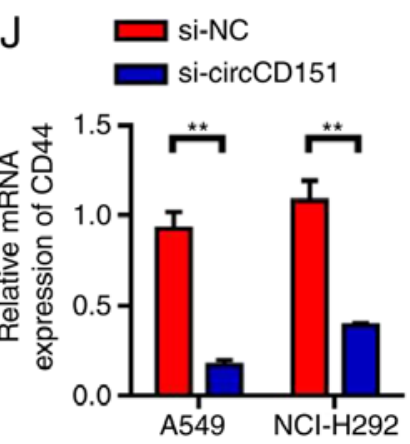

A549

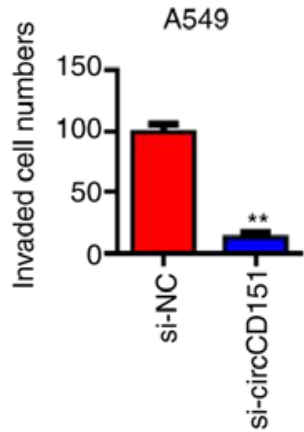

K
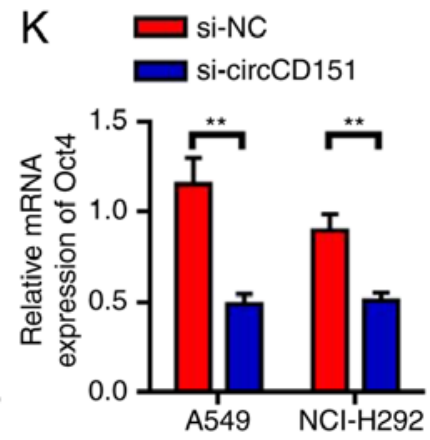

$\mathrm{NCl}-\mathrm{H} 292$

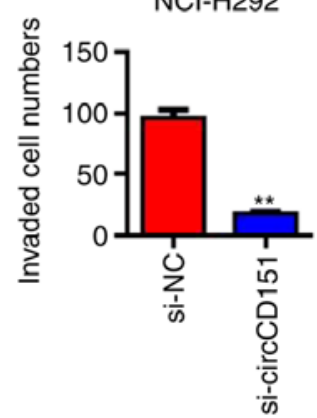

Figure 2. Knockdown of circCD151 inhibits the malignant behavior of lung cancer cells. Detection of circCD151 expression level in (A) A549 and (B) NCI-H292 cell lines. CCK-8 detected the effect of circCD151 on the proliferation of (C) A549 and (D) NCI-H292 cell lines. Clone formation to test the effect of circCD151 on the colony formation ability of (E) A549 and (F) NCI-H292 cell lines. Transwell detection of the influence of circCD151 on the invasion ability of (G) A549 and (H) NCI-H292 cell lines. (I) SOX2, (J) CD44, (K) Oct4 and (L) Nanog expression following circCD151 knockdown in A549 and NCI-H292 cells. Data are presented as the means \pm standard deviation. ${ }^{* *} \mathrm{P}<0.01$. Circ, circular RNA; si, short interfering, NC, negative control.

results demonstrated that GLI2 expression was decreased in A549 and NCI-H292 cells after circCD151 knockdown (Fig. 4H).

circCD151 promotes malignant progression of lung cancer through miR-30d-5p/GLI2 molecular axis. First, RT-qPCR was used to detect the influence of circCD151 overexpression or circCD151+miR-30d-5p overexpression on GLI2 expression. The results demonstrated that the overexpression of circCD151 upregulated GLI2 expression in A549 and NCI-H292 cells compared with the control group. However, following transfection with circCD151+miR-30d-5p mimics, the expression of GLI2 was significantly lower compared with that of circCD151 transfection only (Fig. 5A and B). At 

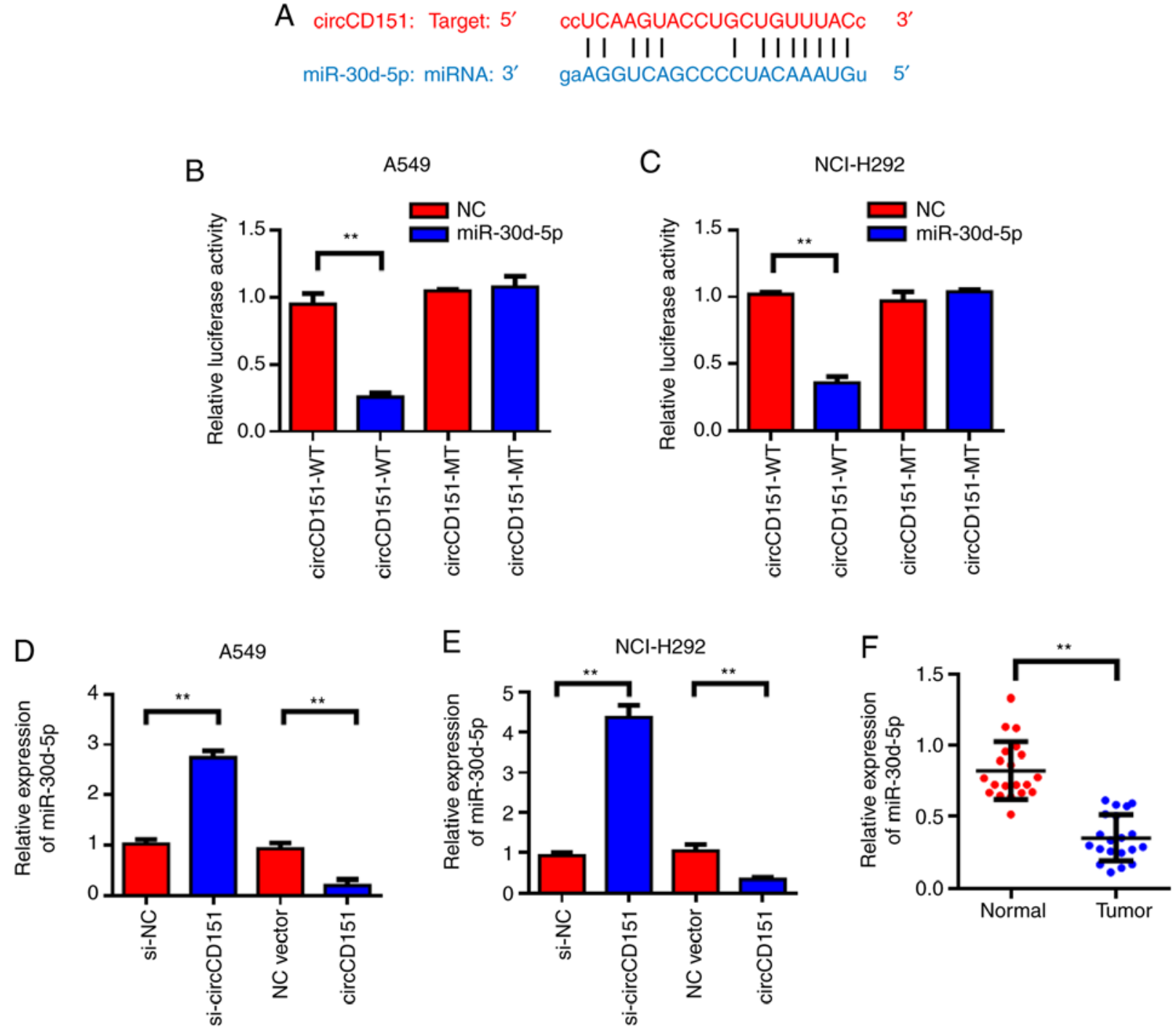

Figure 3. circCD151 targets miR-30d-5p. (A) circCD151 target binding site information for miR-30d-5p. Dual luciferase assay of (B) A549 and (C) NCI-H292 cells. Detection of the effect of circCD151 on the expression level of miR-30d-5p in (D) A549 and (E) NCI-H292 cells. (F) Detection of the expression level of miR-30d-5p in adjacent and lung cancer tissues. Data are presented as the means \pm standard deviation. ${ }^{* *} \mathrm{P}<0.01$. Circ, circular RNA; miR, microRNA; si, short interfering, NC, negative control; WT, wild type; MUT, mutant.

the same time, CCK- 8 was used to detect the proliferation ability of cells. The results demonstrated that overexpression of circCD151 significantly upregulated the proliferation of A549 and NCI-H292 cells. However, after the simultaneous overexpression of circCD151+miR-30d-5p, the cell proliferation ability decreased (Fig. 5C and D). Transwell results demonstrated that overexpression of circCD151 significantly upregulated the invasion ability of A549 and NCI-H292 cells. However, after the simultaneous overexpression of circCD151+miR-30d-5p, the cell invasion ability was reduced (Fig. 5E and F). Therefore, the overexpression of circCD151 promoted the malignant progression of lung cancer by targeting miR-30d-5p and upregulating GLI2.

GLI2 knockdown reverses the oncogenic activity of circCDI51. Verification results of siRNA GLI2 knockdown efficiency is presented in Fig. S2. After the overexpression of circCD151 or circCD151+siGLI2 was detected by RT-qPCR, the expression level of GLI2 was observed to change. The results demonstrated that overexpression of circCD151 could upregulate GLI2 expression. However, following transfection with circCD151+si-GLI2, the expression of GLI2 was significantly decreased (Fig. 6A and B). The proliferation of cells was then tested by CCK-8. The results demonstrated that overexpression of circCD151 significantly upregulated the proliferation of A549 and NCI-H292 cells. However, after circCD151+si-GLI2 was overexpressed at the same time, the proliferation ability of the cells decreased (Fig. 6C and D). Results of clone formation experiments demonstrated that overexpression of circCD151 could increase the clonal formation capacity of A549 and NCI-H292 cells. However, after the simultaneous overexpression of circCD151+si-GLI2, the cell clonal formation ability decreased (Fig. 6E and F). Transwell results demonstrated that overexpression of circCD151 significantly upregulated the invasion ability of A549 and NCI-H292 cells. However, after simultaneous overexpression of circCD151+si-GLI2, cell invasion ability decreased (Fig. 6G and H). Therefore, GLI2 knockdown reduced the oncogenic activity of circCD151. 
A miR-30d-5p: 5' ...GGCCUCUCCAGACCGUGUUUACA...

IIII|

GLI2: $3^{\prime} \quad$ GAAGGUCAGCCCCUACAAAUGU
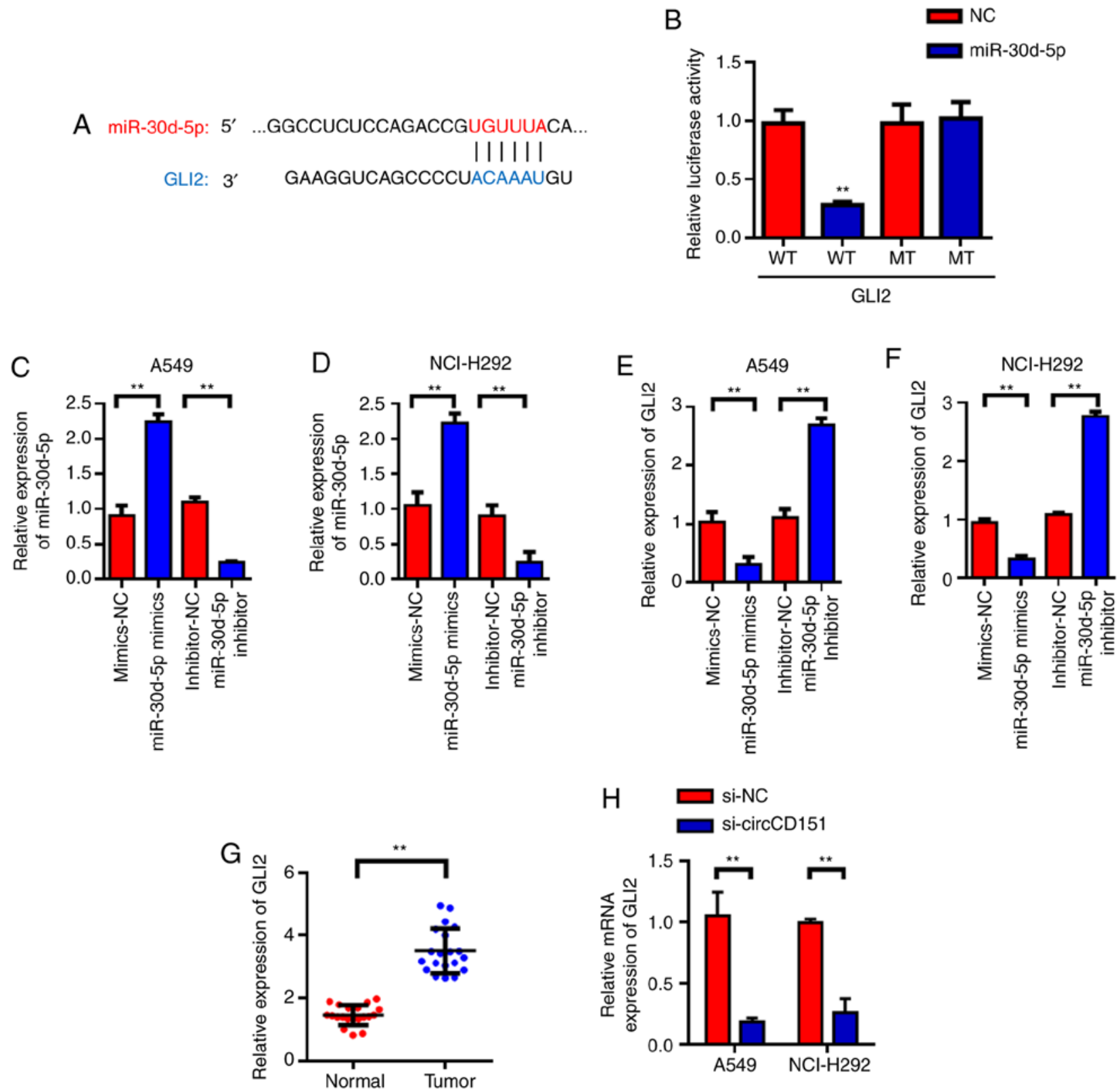

Figure 4. miR-30d-5p targets GLI2. (A) GLI2 locus information for miR-30d-5p targets. (B) Dual luciferase assay. Verification of the transfection efficiency of miR-30d-5p in (C) A549 and (D) NCI-H292 cells. miR-30d-5p inhibits the expression of GLI2 in (E) A549 and (F) NCI-H292 cells. (G) GLI2 reverse transcription-quantitative PCR detection of lung cancer tissues. (H) Detection results of GLI2 expression following circCD151 knockdown in A549 and NCI-H292 cells. Data are presented as the means \pm standard deviation. ${ }^{* *} \mathrm{P}<0.01$. miR, microRNA; Circ, circular RNA; NC, negative control; WT, wild type; MUT, mutant.

\section{Discussion}

CircRNAs, as competitive endogenous RNAs or miRNAs sponges, regulate variable splicing or transcription and the expression of parental genes (30). As miRNAs sponges, circRNAs may serve a major role in the progression of lung cancer by regulating the expression of oncogenes and/or tumor suppressor genes (31). However, little is known about the molecular mechanism of the positive or negative relationship between circRNAs and miRNAs in lung cancer $(32,33)$.

The role of circRNA in lung cancer has been widely studied, but as a biomarker its mechanism of action still need to be further clarified $(34,35)$. Zhu et al (36) first used microarray chip technology to screen circRNA in lung adenocarcinoma tissue and found that the expression of nearly 60 circRNA molecules is changed. It was further confirmed that the upregulation of hsa_circ_0013958 expression is closely related to the proliferation and metastasis of cancer cells. Zhao et al (37) screened 357 differentially expressed circRNAs by sequencing and analysis of tumor samples and adjacent normal tissues of 4 patients with early lung adenocarcinoma.

The present study found that circCD151 was upregulated to different degrees in lung cancer tissues and cell lines by RT-qPCR. circCD151 may serve the role of oncogene in lung cancer and is a potential tumor marker. In this study, we focused on the biological function of the novel circCD151 in lung cancer. The reasons for selecting circCD151 in this study are based on a series of analysis results. The present study 
A
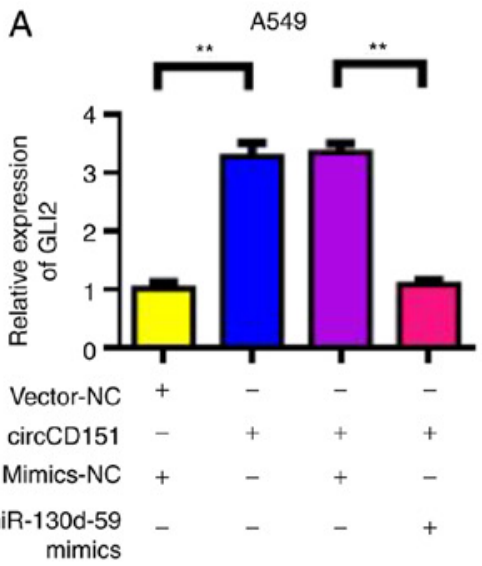

C

A549
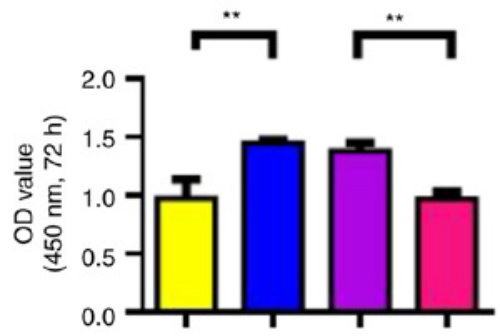

Vector-NC

circCD151

Mimics-NC

miR-130d-59

mimics

E

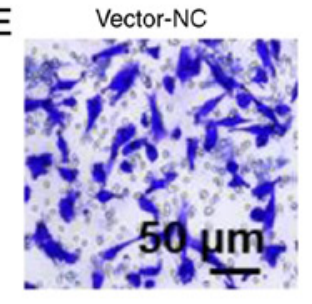

circCD151+

mimics-NC

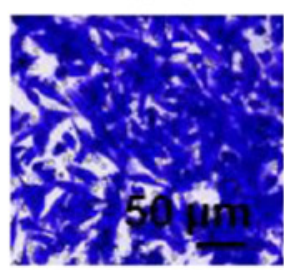

circCD151+

miR-30d-5p mimics
B
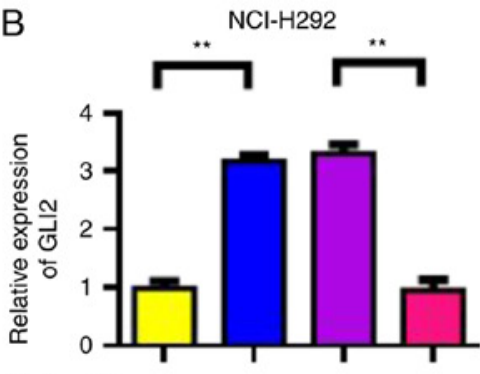

Vector-NC

circCD151

Mimics-NC

miR-130d-59

mimics

D
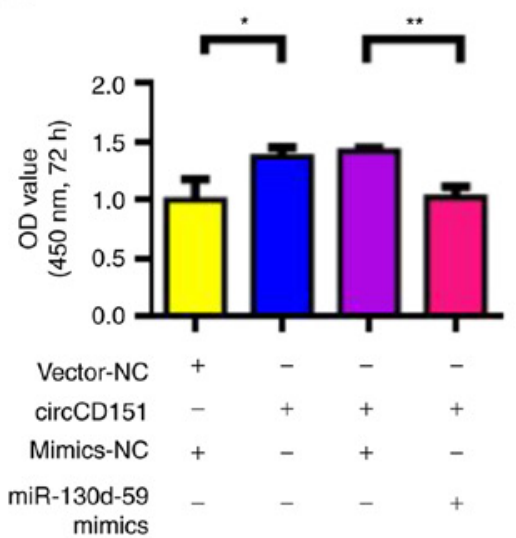

F
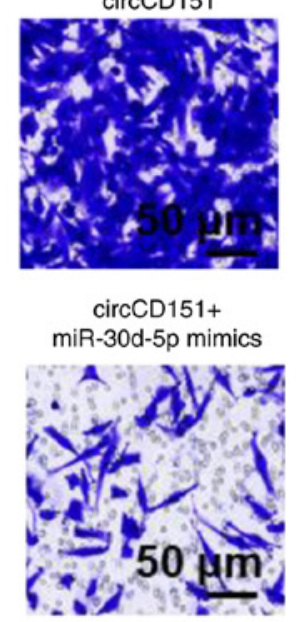

A549

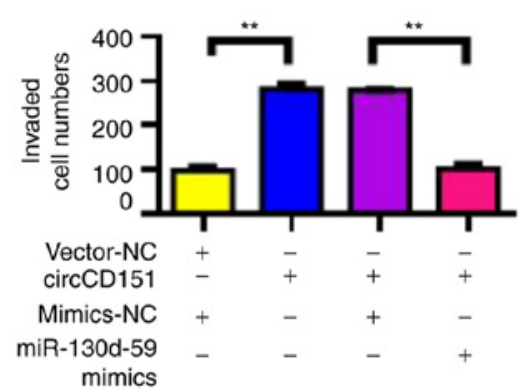

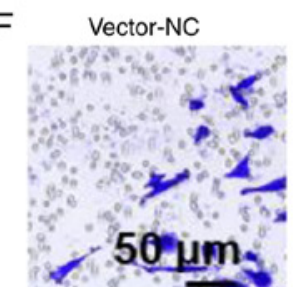

$50 \% \mathrm{~m}$

circCD151+

mimics-NC
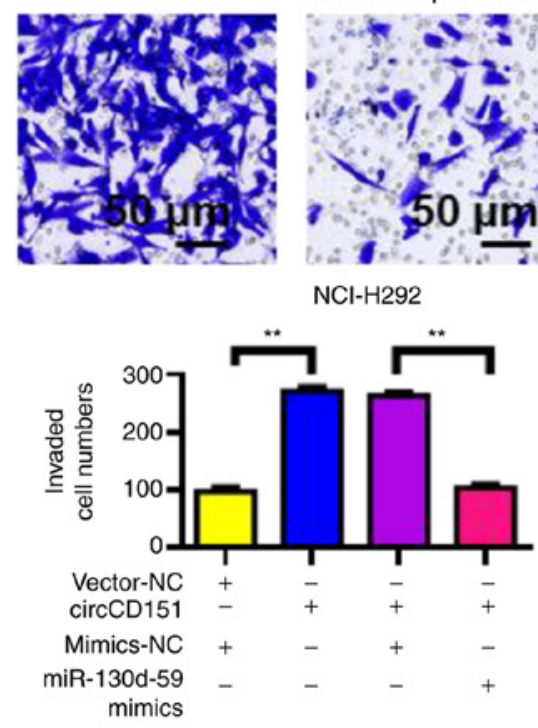

Figure 5. circCD151 promotes the proliferation, migration and invasion of lung cancer through the miR-30d-5p/GLI2 axis. Reverse transcription-quantitative PCR detection of GLI2 expression in (A) A549 and (B) NCI-H292 following overexpression of circCD151 and co-transfection of miR-30d-5p mimics. CCK-8 experiment to detect cell proliferation ability of (C) A549 cells and (D) NCI-H292 cells overexpressing circCD151 and co-transfected with miR-30d-5p mimics. Transwell assay to detect cell invasion ability of (E) A549 and (F) NCI-H292 cells overexpressing circCD151 and co-transfected with miR-30d-5p mimics. Data are presented as the means \pm standard deviation. ${ }^{*} \mathrm{P}<0.05 ;{ }^{* *} \mathrm{P}<0.01$. Circ, circular RNA; miR, microRNA; NC, negative control. 
A

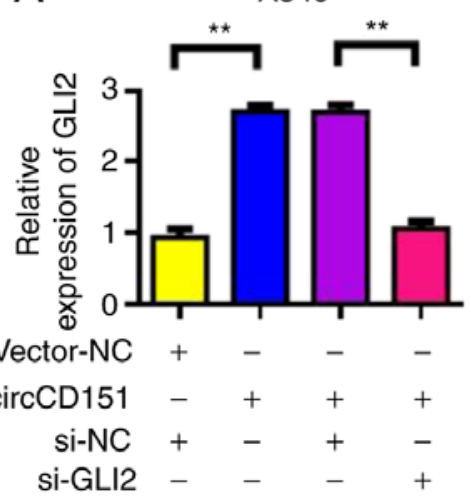

D

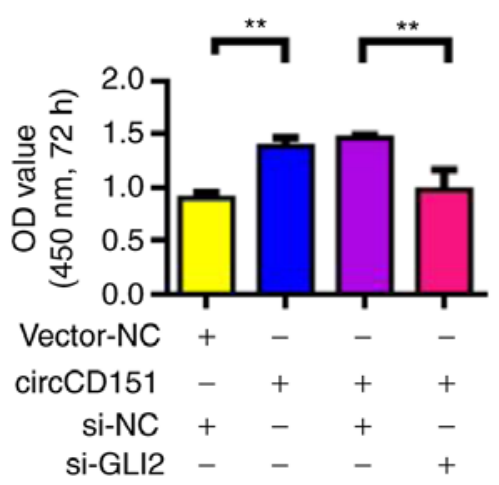

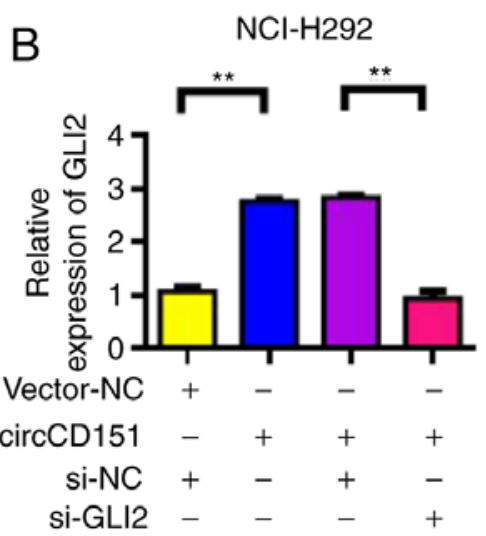

E

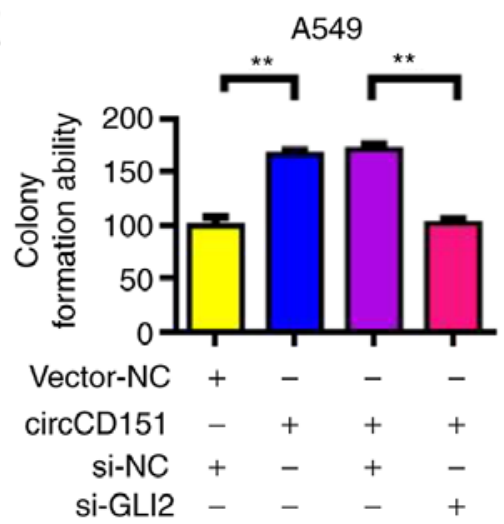

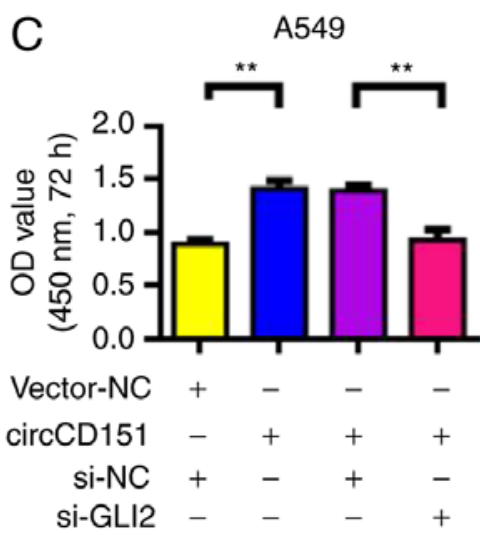

F $\mathrm{NCl}-\mathrm{H} 292$

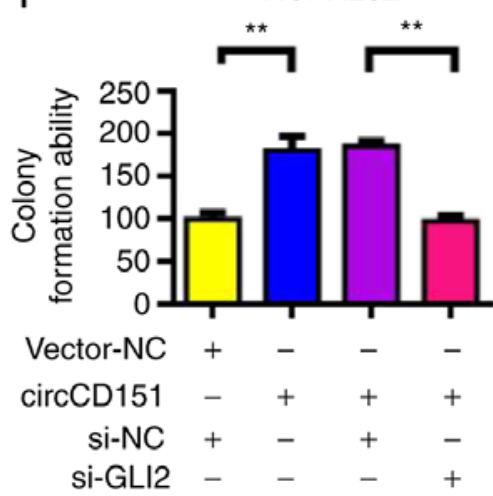

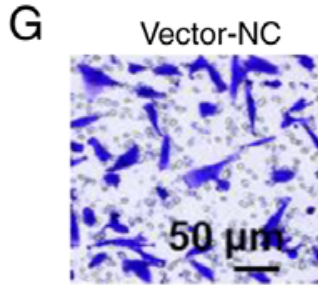

circCD151

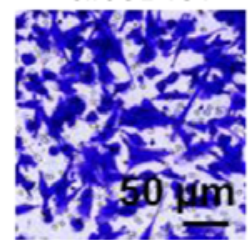

circCD151+ si-NC

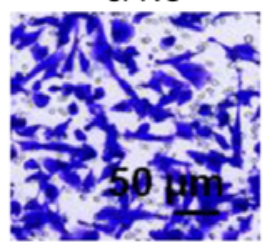

circCD151+

si-GLI2

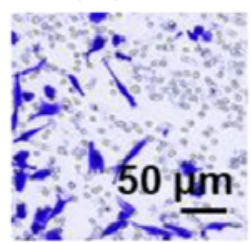

A549

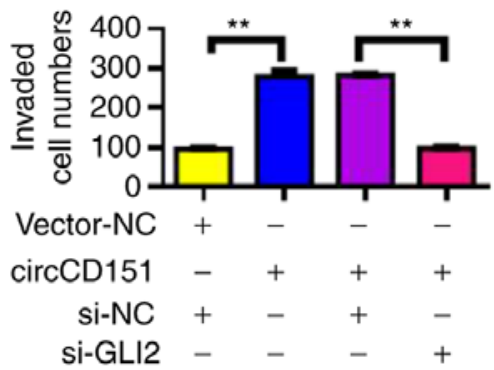

$\mathrm{H}$

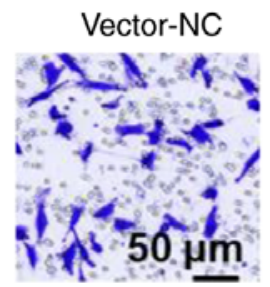

circCD151+ si-NC

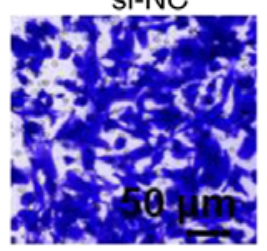

circCD151+ si-GLI2
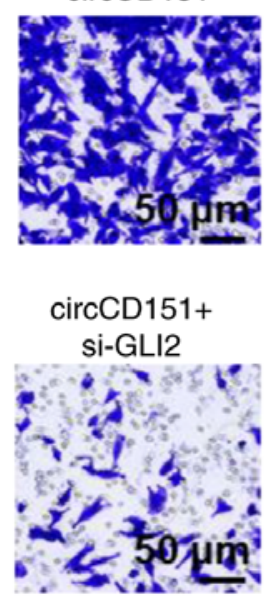

$\mathrm{NCl}-\mathrm{H} 292$

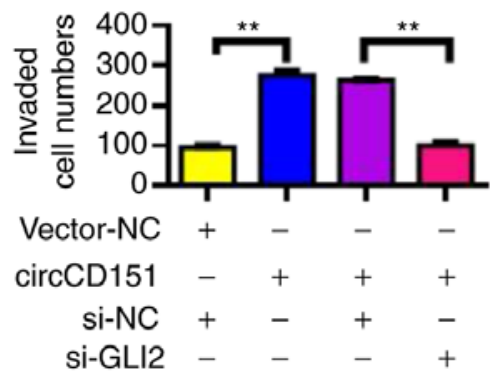

Figure 6. Downregulation of GLI2 reverses the tumor phenotype caused by overexpression of circCD151. A. Reverse transcription-quantitative PCR detection of GLI2 expression in (A) A549 and (B) NCI-H292 following different treatments. CCK-8 detection of the proliferation rate of (C) A549 and (D) NCI-H292 cells following different treatments. Clone formation test the effect of different treatments on the stemness of (E) A549 and (F) NCI-H292 cells. Transwell assay to detect the migration rate of $(\mathrm{G})$ A549 and (H) NCI-H292 cells following different treatments. Data are presented as the means \pm standard deviation. ${ }^{* *} \mathrm{P}<0.01$. Circ, circular RNA; si, short interfering; NC, negative control. 
used StarBase to predict that circCD151 could combine with miR-30d-5p. GLI2 was further predicted to be the target gene of miR-30d-5p by using TargetScan. Finally, dual luciferase reporter gene assay confirmed the existence of the ceRNA regulation mechanism of circCD151/miR-30d-5p/GLI2 in lung cancer.

The present study found that circCD151 was downregulated following knockdown, suggesting that circCD151 may be involved in the malignant progression of lung cancer by regulating GLI2. Furthermore, circCD151 indirectly regulated GLI2 expression through the adsorption of miR-30d-5p. Studies have shown that microRNA serves an important role in the occurrence and development of a variety of tumors, including the invasion and metastasis of tumors (38-40). One of them, miR-30d, has been shown to have a significant anticancer effect. In the tumor tissues of patients with esophageal squamous cell carcinoma, the expression of miR-30d is significantly lower compared with normal tissues. Following the overexpression of miR-30d in tumor cells, the growth, migration and invasion ability of cells were significantly reduced. In addition, miR-30d can also inhibit PI3K/Akt and MEK/ERK signaling pathways, resulting in corresponding biological functions (41-45). In the present study, miR-30d-5p was found to have a tumor suppressive effect. Overexpression of miR-30d-5p could reverse the oncogenic effect of circCD151.

GLI2 is a key factor in Hh signaling mediated tumor proliferation, apoptosis resistance, angiogenesis and invasion and metastasis (46). Studies have shown that by interfering GLI2 expression and downregulating downstream target gene transcription with RNAi technology, the proliferation and metastasis of liver cancer, prostate cancer and basal cell carcinoma can be inhibited (47-49). In the present study, RT-qPCR analysis demonstrated that the expression levels of GLI2 mRNA in lung cancer tissues were significantly higher compared with adjacent tissues. Knockdown of GLI2 reversed the cancer-promoting effect of circCD151. Cyclin D1 is a key protein regulating $\mathrm{G}_{1} / \mathrm{S}$ phase transformation, which can cause DNA replication and division $(50,51)$. Studies have shown that the expression of CyclinD1 and Bax decreases and the expression of Bcl-2 increases following GLI2 gene silencing $(52,53)$. It was hypothesized that the expression of cyclin D1 was decreased after GLI2 silencing and the tumor cells had cycle arrest, which might be in the $G_{1}$ phase, leading to prolonged cell doubling time. The cell cycle was prolonged and the ability to proliferate was decreased. The increased ratio of anti-apoptotic Bcl-2 to pro-apoptotic Bax induces downstream apoptotic cascade effect, promotes cell apoptosis and ultimately leads to weakened cell growth ability (54).

The findings of the present study and the investigation of the function of circCD151, may provide further reference for the treatment of lung cancer. The advancement of gene therapy vectors and the development of pharmaceutical engineering are expected to develop a highly effective and feasible intervention against circCD151. Further research directions include the development of novel non-viral gene vectors with higher transfection efficiency.

There are also shortcomings in the present study. The expression of circRNA is regulated by a variety of factors. The present study only observed that circCD151 was highly expressed in lung cancer cells and tissues, but the upstream regulatory factor of circCD151 remains to be elucidated. As an important pro-cancer factor, GLI2 serves an important role in the development of lung cancer and the downstream regulatory signaling pathway of GLI2 also requires further study.

In summary, circCD151 was significantly increased in lung cancer tissues and cells. Silencing the circCD151 gene in A549 and NCI-H292 cells inhibited the proliferation, migration and invasion of A549 and NCI-H292 cells. The upregulated expression of circCD151 could promote the proliferation, migration and invasion of A549 and NCI-H292 cells. These results indicated that circCD151 serves an important role in the development and progression of lung cancer. Mechanism studies showed that circCD151 promoted cancer by regulating miR-30d-5p/GLI2 axis.

\section{Acknowledgements}

Not applicable.

Funding

No funding was received.

\section{Availability of data and materials}

The datasets used and/or analyzed in the current study are available from the corresponding author on reasonable request.

\section{Authors' contributions}

LZ and PJ confirm the authenticity of all the raw data. PJ designed the experiments. LZ and $\mathrm{HZ}$ performed the experiments and data analysis. LZ wrote the manuscript, with contributions from all authors. All authors read and approved the final manuscript.

\section{Ethics approval and consent to participate}

The present study was approved by the medical Ethics Committee of Tianjin First Central Hospital (approval no. TJ202008096). All patients signed informed consent.

\section{Patient consent for publication}

Not applicable.

\section{Competing interests}

The authors declare that they have no competing interests.

\section{References}

1. de Groot PM, Wu CC, Carter BW and Munden RF: The epidemiology of lung cancer. Transl Lung Cancer Res 7: 220-233, 2018.

2. Barta JA, Powell CA and Wisnivesky JP: Global epidemiology of lung cancer. Ann Glob Health 85: 8, 2019.

3. Herbst RS, Morgensztern D and Boshoff C: The biology and management of non-small cell lung cancer. Nature 553: 446-454, 2018.

4. Hirsch FR, Scagliotti GV, Mulshine JL, Kwon R, Curran WJ Jr, Wu YL and Paz-Ares L: Lung cancer: Current therapies and new targeted treatments. Lancet 389: 299-311, 2017. 
5. Xi X, Liu N, Wang Q, Chu Y, Yin Z, Ding Y and Lu Y: ACT001, a novel PAI-1 inhibitor, exerts synergistic effects in combination with cisplatin by inhibiting PI3K/AKT pathway in glioma. Cell Death Dis 10: 757, 2019.

6. Zhong W, Yang W, Qin Y, Gu W, Xue Y, Tang Y, Xu H, Wang $\mathrm{H}$, Zhang $\mathrm{C}$, Wang $\mathrm{C}$, et al: 6-Gingerol stabilized the p-VEGFR2/VE-cadherin/ $\beta$-catenin/actin complex promotes microvessel normalization and suppresses tumor progression. J Exp Clin Cancer Res 38: 285, 2019.

7. Salzman J, Gawad C, Wang PL, Lacayo N and Brown PO: Circular RNAs are the predominant transcript isoform from hundreds of human genes in diverse cell types. PLoS One 7: e30733, 2012.

8. Chen D, Ma W, Ke Z and Xie F: CircRNA hsa_circ 100395 regulates miR-1228/TCF21 pathway to inhibit lung cancer progression. Cell Cycle 17: 2080-2090, 2018.

9. Zhang HD, Jiang LH, Sun DW, Hou JC and Ji ZL: CircRNA: A novel type of biomarker for cancer. Breast Cancer 25: 1-7, 2018.

10. Jiang MM, Mai ZT, Wan SZ, Chi YM, Zhang X, Sun BH and Di QG: Microarray profiles reveal that circular RNA hsa_ circ_0007385 functions as an oncogene in non-small cell lung cancer tumorigenesis. J Cancer Res Clin Oncol 144: 667-674, 2018 .

11. Zhang S, Zeng X, Ding T, Guo L, Li Y, Ou S and Yuan H: Microarray profile of circular RNAs identifies hsa circ 0014130 as a new circular RNA biomarker in non-small cell lung cancer. Sci Rep 8: 2878, 2018

12. Yao JT, Zhao SH, Liu QP, Lv MQ, Zhou DX, Liao ZJ and Nan KJ: Over-expression of CircRNA_100876 in non-small cell lung cancer and its prognostic value. Pathol Res Pract 213: 453-456, 2017.

13. Wei CY, Zhu MX, Lu NH, Liu JQ, Yang YW, Zhang Y, Shi YD, Feng ZH, Li JX, Qi FZ and Gu JY: Circular RNA circ 0020710 drives tumor progression and immune evasion by regulating the miR-370-3p/CXCL12 axis in melanoma. Mol Cancer 19: 84, 2020.

14. Ambros V: MicroRNAs: Tiny regulators with great potential Cell 107: 823-826, 2001.

15. Xi X, Chu Y, Liu N, Wang Q, Yin Z, Lu Y and Chen Y: Joint bioinformatics analysis of underlying potential functions of hsa-let-7b-5p and core genes in human glioma. J Transl Med 17: 129,2019

16. de Moor CH, Meijer H and Lissenden S: Mechanisms of translational control by the 3' UTR in development and differentiation. Semin Cell Dev Biol 16: 49-58, 2005

17. Bartel DP: MicroRNAs: Genomics, biogenesis, mechanism, and function. Cell 116: 281-297, 2004.

18. Manikandan J, Aarthi JJ, Kumar SD and Pushparaj PN: Oncomirs: The potential role of non-coding microRNAs in understanding cancer. Bioinformation 2: 330-334, 2008

19. Esquela-Kerscher A and Slack FJ: Oncomirs-microRNAs with a role in cancer. Nat Rev Cancer 6: 259-269, 2006

20. Zhang B, Pan X, Cobb GP and Anderson TA: MicroRNAs as oncogenes and tumor suppressors. Dev Biol 302: 1-12, 2007.

21. Waldman SA and Terzic A: MicroRNA signatures as diagnostic and therapeutic targets. Clin Chem 54: 943-944, 2008.

22. Sicklick JK, Li YX, Jayaraman A, Kannangai R, Qi Y, Vivekanandan P, Ludlow JW, Owzar K, Chen W, Torbenson MS and Diehl AM: Dysregulation of the Hedgehog pathway in human hepatocarcinogenesis. Carcinogenesis 27: 748-757, 2006.

23. Patil MA, Zhang J, Ho C, Cheung ST, Fan ST and Chen X: Hedgehog signaling in human hepatocellular carcinoma. Cancer Biol Ther 5: 111-117, 2006.

24. Huang S, He J, Zhang X, Bian Y, Yang L, Xie G, Zhang K, Tang W, Stelter AA, Wang Q, et al: Activation of the hedgehog pathway in human hepatocellular carcinomas. Carcinogenesis 27 : 1334-1340, 2006.

25. Tang YA, Chen YF, Bao Y, Mahara S, Yatim SMJM, Oguz G Lee PL, Feng M, Cai Y, Tan EY, et al: Hypoxic tumor microenvironment activates GLI2 via HIF- $1 \alpha$ and TGF- $\beta 2$ to promote chemoresistance in colorectal cancer. Proc Natl Acad Sci USA 115: E5990-E5999, 2018

26. Xia L, Bouamar H, Gu X, Zeballos C, Qin T, Wang B, Zhou Y, Wang Y, Yang J, Zhu H, et al: Gli2 mediates the development of castration-resistant prostate cancer. Int J Oncol 57: 100-112, 2020.

27. Detterbeck FC, Boffa DJ, Kim AW and Tanoue LT: The eighth edition lung cancer stage classification. Chest 151: 193-203, 2017.
28. Koul R, Rathod S, Dubey A, Bashir B and Chowdhury A: Comparison of $7 \mathrm{th}$ and 8th editions of the UICC/AJCC TNM staging for non-small cell lung cancer in a non-metastatic North American cohort undergoing primary radiation treatment. Lung Cancer 123: 116-120, 2018.

29. Livak KJ and Schmittgen TD: Analysis of relative gene expression data using real-time quantitative PCR and the 2(-Delta Delta C(T)) method. Methods 25: 402-408, 2001

30. Hu W, Bi ZY, Chen ZL, Liu C, Li LL, Zhang F, Zhou Q, Zhu W, Song YY, Zhan BT, et al: Emerging landscape of circular RNAs in lung cancer. Cancer Lett 427: 18-27, 2018.

31. Huang X, Zhang W and Shao Z: Prognostic and diagnostic significance of circRNAs expression in lung cancer. J Cell Physiol 234: 18459-18465, 2019.

32. Ma Y, Zhang X, Wang YZ, Tian $\mathrm{H}$ and Xu S: Research progress of circular RNAs in lung cancer. Cancer Biol Ther 20: 123-129, 2019.

33. Di X, Jin X, Li R, Zhao M and Wang K: CircRNAs and lung cancer: Biomarkers and master regulators. Life Sci 220: 177-185, 2019.

34. Wang C, Tan S, Li J, Liu WR, Peng Y and Li W: CircRNAs in lung cancer-Biogenesis, function and clinical implication. Cancer Lett 492: 106-115, 2020.

35. Braicu C, Zimta AA, Harangus A, Iurca I, Irimie A, Coza O and Berindan-Neagoe I: The function of non-coding RNAs in lung cancer tumorigenesis. Cancers (Basel) 11: 605, 2019.

36. Zhu X, Wang X, Wei S, Chen Y, Chen Y, Fan X, Han S and Wu G: hsa_circ_0013958: A circular RNA and potential novel biomarker for lung adenocarcinoma. FEBS J 284: 2170-2182, 2017.

37. Zhao J, Li L, Wang Q, Han H, Zhan Q and Xu M: CircRNA expression profile in early-stage lung adenocarcinoma patients. Cell Physiol Biochem 44: 2138-2146, 2017.

38. McGuire A, Brown JA and Kerin MJ: Metastatic breast cancer: The potential of miRNA for diagnosis and treatment monitoring. Cancer Metastasis Rev 34: 145-155, 2015.

39. van Schooneveld E, Wildiers H, Vergote I, Vermeulen PB, Dirix LY and Van Laere SJ: Dysregulation of microRNAs in breast cancer and their potential role as prognostic and predictive biomarkers in patient management. Breast Cancer Res 17: 21, 2015.

40. Zhong W, Hou H, Liu T, Su S, Xi X, Liao Y, Xie R, Jin G, Liu X, Zhu L, et al: Cartilage oligomeric matrix protein promotes epithelial-mesenchymal transition by interacting with transgelin in colorectal cancer. Theranostics 10: 8790-8806, 2020.

41. Chung SJ, Nagaraju GP, Nagalingam A, Muniraj N, Kuppusamy P, Walker A, Woo J, Győrffy B, Gabrielson E, Saxena NK and Sharma D: ADIPOQ/adiponectin induces cytotoxic autophagy in breast cancer cells through STK11/LKB1-mediated activation of the AMPK-ULK1 axis. Autophagy 13: 1386-1403, 2017.

42. Cheng SM, Chang YC, Liu CY, Lee JY, Chan HH, Kuo CW, Lin KY, Tsai SL, Chen SH, Li CF, et al: YM155 down-regulates survivin and XIAP, modulates autophagy and induces autophagy-dependent DNA damage in breast cancer cells. Br J Pharmacol 172: 214-234, 2015.

43. Zhu B, Chen H, Zhang X, Pan Y, Jing R, Shen L, Wang X, Ju S, Jin C and Cong H: Serum miR-30d as a novel biomarker for multiple myeloma and its antitumor role in U266 cells through the targeting of the MTDH/PI3K/Akt signaling pathway. Int J Oncol 53: 2131-2144, 2018.

44. Xu X, Zong K, Wang X, Dou D, Lv P, Zhang Z and Li H: MiR-30d suppresses proliferation and invasiveness of pancreatic cancer by targeting the SOX4/PI3K-AKT axis and predicts poor outcome. Cell Death Dis 12: 350, 2021

45. Ye C, Yu X, Liu X, Dai M and Zhang B: MiR-30d inhibits cell biological progression of Ewing's sarcoma by suppressing the MEK/ERK and PI3K/Akt pathways in vitro. Oncol Lett 15: 4390-4396, 2018

46. Bhateja P, Cherian M, Majumder S and Ramaswamy B: The Hedgehog signaling pathway: A viable target in breast cancer? Cancers (Basel) 11: 1126, 2019.

47. He Y, Huang H, Jin L, Zhang F, Zeng M, Wei L, Tang S, Chen D and Wang W: CircZNF609 enhances hepatocellular carcinoma cell proliferation, metastasis, and stemness by activating the Hedgehog pathway through the regulation of miR-15a-5p/15b-5p and GLI2 expressions. Cell Death Dis 11: 358, 2020.

48. Thiyagarajan S, Bhatia N, Reagan-Shaw S, Cozma D, Thomas-Tikhonenko A, Ahmad N and Spiegelman VS: Role of GLI2 transcription factor in growth and tumorigenicity of prostate cells. Cancer Res 67: 10642-10646, 2007. 
49. Regl G, Neill GW, Eichberger T, Kasper M, Ikram MS, Koller J, Hintner H, Quinn AG, Frischauf AM and Aberger F: Human GLI2 and GLI1 are part of a positive feedback mechanism in basal cell carcinoma. Oncogene 21: 5529-5539, 2002.

50. Hinz M, Krappmann D, Eichten A, Heder A, Scheidereit C and Strauss M: NF-kappaB function in growth control: Regulation of cyclin D1 expression and G0/G1-to-S-phase transition. Mol Cell Biol 19: 2690-2698, 1999.

51. Saha A, Halder S, Upadhyay SK, Lu J, Kumar P, Murakami M, Cai $Q$ and Robertson ES: Epstein-Barr virus nuclear antigen 3C facilitates G1-S transition by stabilizing and enhancing the function of cyclin D1. PLoS Pathog 7: e1001275, 2011.

52. Rutter M, Wang J, Huang Z, Kuliszewski M and Post M: Gli2 influences proliferation in the developing lung through regulation of cyclin expression. Am J Respir Cell Mol Biol 42: 615-625, 2010.
53. Zhang D, Liu J, Wang Y, Chen J and Chen T: shRNA-mediated silencing of Gli2 gene inhibits proliferation and sensitizes human hepatocellular carcinoma cells towards TRAIL-induced apoptosis. J Cell Biochem 112: 3140-3150, 2011.

54. Singh R, Letai A and Sarosiek K: Regulation of apoptosis in health and disease: The balancing act of BCL-2 family proteins. Nat Rev Mol Cell Biol 20: 175-193, 2019.

cc) (i) (2) This work is licensed under a Creative Commons Attribution-NonCommercial-NoDerivatives 4.0 International (CC BY-NC-ND 4.0) License. 